Artículo original

Factores de riesgo cardiovascular y síndrome metabólico en pacientes con lupus eritematoso sistémico

\title{
Cardiovascular Risk Factors and Metabolic Syndrome in Patients with SLE
}

Dora Elizabeth Montiel de Jarolin ${ }^{1}$

${ }^{1}$ Ministerio de Salud Pública y Bienestar Social, Hospital Nacional, Departamento de Medicina Interna. Itauguá, Paraguay.

\section{RESUMEN}

Introducción:La aterosclerosis acelerada que ocurre en el LES se considera uno de los mecanismos fundamentales del aumento de la mortalidad de causa cardiovascular. Entre los factores que contribuyen al proceso de la aterosclerosis acelerada se incluyen factores de riesgo cardiovascular tradicionales y factores relacionados con el LES.

Objetivo: identificar los factores de riesgo cardiovasculares relacionados con el LES y los factores de riesgo tradicionales asociados en pacientes con LES.

Material y método: estudio descriptivo de corte trasverso con componente analítico de pacientes con diagnóstico de LES que acudieron al Hospital Día del Departamento de Medicina Interna del Hospital Nacional de enero 2015 a marzo 2016. Las variables analizadas fueron: edad, procedencia, sexo, tiempo de diagnóstico del LES, actividad de la enfermedad, presencia de nefritis lúpica, nefropatía crónica, sobrepeso, obesidad, cintura en $\mathrm{cm}$, antecedentes de sedentarismo, tabaquismo, hipertensión arterial (HTA), HTA controlada, hipercolesterolemia, hipertigliceridemia, HDL bajo, síndrome metabólico, anticuerpoanticardiolipina. Para el componente analítico se utilizó la prueba del Chi cuadrado y regresión logística para establecer asociación entre las variables.

Resultados: fueron estudiados 114 pacientes, siendo 96 del sexo femenino $(84,2 \%)$ y 18 del masculino $(16 \%)$. La edad media fue $36 \pm 14$ años. Procedían del Departamento Central 42 (42\%) e interior 59 (58\%). El tiempo medio de diagnóstico de LES fue $5 \pm 6$ años. Se halló LES activo $40(35 \%)$, nefritis lúpica $46(40,4 \%)$, sobrepeso $43(38 \%)$, obesidad 18 $(16 \%)$, sedentarismo $52(46 \%)$, HTA $47(42 \%)$, con HTA controlada 24 $(24 \%)$, tabaquismo $3(3 \%)$, etilismo $3(3 \%)$. Tenían hipercolesterolemia $38(33,8 \%)$, hipertrigliceridemia $24(21,1 \%)$ c-HDL bajo $44(40 \%)$. La frecuencia de síndrome metabólico fue $2 \%$. Ac anticardiolipinafue positivo en 1 , C3 y C4 aumentado en $5(4,4 \%)$ y nefropatía crónica $11(10 \%)$. La dosis media de prednisona fue $25 \mathrm{mg} /$ día. Hubo 1 infarto agudo de miocardio, elelectrocardiograma (ECG) fue anormal en 5 y ecocardiografía anormal en 14 casos. La nefritis lúpica se asoció significativamente con el 
desarrollo de HTA (p 0,05).

Conclusión: los principales factores de riesgo vascular relacionados con el LES fueron: actividadde la enfermedad, tiempo de enfermedad, nefritis lúpica. Los principales factores de riesgo vascular tradicionales identificados fueron: HTA, sobrepeso/obesidad, CHDL bajo, sedentarismo, dislipidemia. La frecuencia del síndrome metabólico fue bajo (2\%). La presencia de nefritis lúpica se relacionó en forma estadísticamente significativa con el desarrollo de HTA.

Palabras clave: lupus eritematoso sistémico, factores de riesgo cardiovascular, síndrome metabólico.

\section{ABSTRACT}

Introduction: accelerated atherosclerosis that occurs in SLE is considered one of the principal mechanisms of increased mortality due to cardiovascular causes. Between factors which contribute to the process of accelerated atherosclerosis include traditional cardiovascular risk factors and factors related to SLE.

Objective: to identify the cardiovascular risk factors related to SLE and the associated traditional risk factors in patients with SLE.

Material and method: descriptive study of transverse section with analytical component of patients diagnosed with SLE who attended to Day Hospital of the Department of Internal Medicine of the National Hospital from January 2015 to March 2016. The variables analyzed were: age, origin, sex, time of diagnosis of SLE, activity of the disease, presence of lupus nephritis, chronic nephropathy, overweight, obesity, waist in $\mathrm{cm}$, history of sedentary lifestyle, smoking, hypertension (HBP), controlled hypertension, hypercholesterolemia, hypertiglyceridemia, low HDL, metabolic syndrome, antibody anticardiolipin. For the analytical component, the Chi square test and logistic regression were used to establish an association between the variables.

Results: 114 patients were studied, 96 being female $(84.2 \%)$ and 18 male $(16 \%)$. The average age was $36 \pm 14$ years. They came from the Central Department $42(42 \%)$ and inland $59(58 \%)$. The mean time of diagnosis of SLE was $5 \pm 6$ years. We found active SLE in $40(35 \%)$, lupus nephritis $46(40.4 \%)$, overweight $43(38 \%)$, obesity $18(16 \%)$, sedentary lifestyle $52(46 \%)$, HTA $47(42 \%)$, HBP controlled $24(24 \%)$, smoking $3(3 \%)$, alcoholism $3(3 \%)$. They had hypercholesterolemia 38 (33.8\%), hypertriglyceridemia $24(21.1 \%)$ c-HDL low $44(40 \%)$. The frequency of metabolic syndrome was $2 \%$. Ac anticardiolipin was positive in 1, C3 and C4 increased by $5(4.4 \%)$ and nephropathy in disease 11 $(10 \%)$. The average dose of prednisone was $25 \mathrm{mg} /$ day. There was 1 acute myocardial infarction, elelectrocardiogram (ECG) was abnormal in 5 and abnormal echocardiography in 14 cases. Lupus nephritis was significantly associated with the development of hypertension ( $p$ 0.05).

Conclusion: the main vascular risk factors related to SLE were: activity of the disease, time of illness, lupus nephritis. The main traditional vascular risk factors identified were: hypertension, overweight / obesity, low HDL-C, sedentary lifestyle, dyslipidemia. The frequency of the metabolic syndrome was low (2\%). The presence of lupus nephritis was 
related in a statistically significant way with the development of hypertension.

Keyword: systemic lupus erythematosus, cardiovascular risk factors, metabolic syndrome.

\section{Autor correpondiente}

Dra. Dora Elizabeth Montiel de Jarolín. Ministerio de Salud Pública y Bienestar Social, Hospital Nacional, Departamento de Medicina Interna. Itauguá, Paraguay.

Correo electrónico: dradoramontiel@hotmail.com

Artículo recibido: 5 diciembre 2017

Artículo aprobado: 8 mayo 2018

\section{INTRODUCCIÓN}

EI LES es una enfermedad inflamatoria crónica autoinmune que puede presentar complicaciones multisistémicas.Se caracteriza por la activación de linfocitos $\mathrm{T}$ y $\mathrm{B}$ policlonales, producción de autoanticuerpos y la formación de complejos inmunes que ocasionan injuria en tejidos y órganos ${ }^{(1-3)}$.

Con respecto a las complicaciones asociadas a esta patología, las enfermedades cardiovasculares han surgido como una principal causa de morbimortalidad en pacientes con LES, debido a un acelerado desarrollo de ateroesclerosis, como también a un elevado riesgo de presentar eventos aterotrombóticos. La prevalencia de eventos cardiovasculares secundarios a la aterotrombosis en estos pacientes esde $14,07 \%$, de la cual $7,04 \%$ corresponden a la angina de pecho,4,22\% al infarto agudo de miocardio y $2,81 \%$ a los accidentes cerebrovasculares ${ }^{(3-9)}$.

La aterosclerosis acelerada que ocurre en el LES se considera uno de los mecanismos fundamentales del aumento de la mortalidad por enfermedad cardiovascular(10-14). Los pacientes con lupus presentan una elevada prevalencia de HTA $(11,5-75 \%)$, dislipidemia y de síndromemetabólico ${ }^{(15-}$ 19).

La alteración de algunos factores de riesgo cardiovascularesclásicos no pueden explicar en ciertos casos el incremento de riesgo que presentan estos pacientes para sufrir algún evento cardiovascular. Hay otros factores que se deben tomar en cuenta, tales como, tiempo de evolución de la enfermedad, menopausia temprana, uso de fármacos (corticosteroides), daño de la función renal, resistencia a la insulina, hiperhomocisteínemia, incremento de los niveles de proteína C-reactiva, concentración plasmática elevada de fibrinógeno, presencia de inmunocomplejos circulantes $\mathrm{y} / \mathrm{o}$ de proteínas del complemento encontrados en esta enfermedad(6).

La disfunción endotelial juega un papel muy importante en el desarrollo de la aterosclerosis acelerada en pacientes con LES. La evidencia sugiere que el LES acelera el daño vascular y que además los mecanismos para su reparación son inefectivos. Se han observado niveles elevados de células endoteliales apoptóticas circulantes, expresión de daño vascular y niveles bajos de células endoteliales progenitoras encargadas de reparar el daño arterial en sangre de pacientes con LES. Entre los procesos que 
intervienen en el daño endotelial destaca la formación de inmunocomplejos y la activación del complemento, características ambas del LES, así como el interferón alfa, que juega un papel importante tanto en el daño endotelial como en su reparación $(8,9)$.

El objetivo de este estudio fue identificar los factores que se asocian al riesgocardiovascular en los pacientes adultos con LES, tanto los factores de riesgo cardiovascular tradicionales como los relacionados con la enfermedad atendidos en la consulta del Hospital Día del Departamento de Medicina Interna del Hospital Nacional de Itauguá.

\section{MATERIAL Y MÉTODOS}

Estudio descriptivo prospectivo de corte trasverso con componente analítico de 101 pacientes con diagnóstico de LES que acudieron a la consulta del Hospital Día del Departamento de Medicina Interna del Hospital Nacional en el periodo enero 2015a marzo 2016. Fueron incluidos todos los pacientes adultos con diagnóstico de LES de ambos sexos y fueron excluidos los pacientes con otros diagnósticos de enfermedad autoinmune. Las variables analizadas fueron: demográficas, procedencia, el tiempo de enfermedad, la dosis de prednisona recibida, la presencia en el momento del estudio de : lupus activo, nefritis lúpica, nefropatía crónica, la cintura, el índice de masa corporal, el título de anticuerpo antinuclear, título de anticuerpo anti DNA, presencia de hipercomplementemia, presencia de anticuerpo anti cardiolipina y anticoagulante lúpico, los factores de riesgo cardiovascular asociados: HTA, HTA no controlada, sedentarismo, sobrepeso, obesidad, tabaquismo, cHDL bajo, colesterol total, triglicéridos, cLDL. Además: hallazgos del electrocardiograma, ecocardiografía y de la ecografía Doppler de carótida. El diagnóstico de síndrome metabólico fue establecido según los criterios del ATP III.

\section{Definiciones}

Se consideró como diagnóstico de LES cumplir al menos 4 criterios de forma simultánea o consecutiva a lo largo del seguimiento según los Criterios para la clasificación del lupus eritematoso sistémico (American College of Rheumatology - ACR- 1997/Systemic lupus International CollaboratingClinicsGroup-SLICC- 2012)(1).

Se consideró LES activo: por actividad un escenario clínico en el que la enfermedad no está adecuadamente controlada, lo que se traduce en diferentes manifestaciones clínicasy/o analíticas: SLEDAI $\geq 4$ (1)

Se consideró como nefritis lúpica: todo paciente con manifestaciones clínicas de glomerulonefritis aguda con orina simple con proteinuria, sedimento urinario con leucocitaria, hematuria(2).

Se consideró como nefropatía crónica: todo paciente con clereance de creatinina $<60 \mathrm{~mL}$ por minuto del filtrado glomerular normal mantenido durante al menos 3 meses ${ }^{(10)}$.

Se consideró como hipertensión arterial: todo paciente con cifras de presión $\geq 140 / 90 \mathrm{~mm} \mathrm{Hg}{ }^{(11)}$.

Se consideró como hipertensión arterial no controlada: todo paciente con cifras tensionales por encima de los valores considerados normales bajo tratamiento con fármacos hipotensores ${ }^{(11)}$.

Indice de masa corporal (IMC): peso en kilogramos dividido por el cuadrado 
de la talla en metros. Se clasificaron los pacientes como peso normal, sobrepeso u obeso, según la clasificación de la $\mathrm{OMS}^{(12)}$.

Se realizó la medición de circunferencia de la cintura en centímetros considerándose como elevada en mujeres $\geq 88 \mathrm{~cm}$ y en varones $\geq$ $102 \mathrm{~cm}^{(16)}$.

Se consideró hipercolesterolemia a los valores de colesterol total igual o mayor de $\geq 200 \mathrm{mg} / \mathrm{dL}$, hipertrigliceridemia a triglicéridos $\geq 150 \mathrm{mg} / \mathrm{dL}$ y colesterol HDL bajo cuando fue $<40 \mathrm{mg} / \mathrm{dL}$ en varones $<$ de $50 \mathrm{mg} / \mathrm{dL}$ en mujeres(14).

Se estableció el diagnóstico de síndrome metabólico según los criterios del ATP III ${ }^{(16)}$.

\section{Estimación del tamaño de muestra:}

Se utilizó el programa estadístico Epi Info $7^{\circ}$. Para el cálculo del tamaño de la muestra se esperó una proporción de 1,5\% de síndrome metabólico. Con una amplitud de 1,9\%(precisión), nivel de confianza $95 \%$, lo que arrojó un tamaño mínimo de 88 pacientes sobre un universo de 200 pacientes del Hospital de Día.

\section{Análisis de datos:}

Los datos se registraron en planilla electrónica Microsoft EXCEL, posteriormente procesadas con Epi Info $7^{\circ}$ utilizando estadística descriptiva. Los resultados se expresaron en forma deproporciones para las variables cualitativas y como media y desviación estándar para las variables continuas. Se utilizaron tablas para la presentación de los factores de riesgo clínicos y laboratoriales de los pacientes que conformaron la muestra. Para establecer asociaciones entre las variables se utilizaron tablas de contingencia y la prueba del chi cuadrado para variables cualitativas.

\section{RESULTADOS}

Fueron estudiados 114 pacientes con diagnóstico de LES, siendo del sexo femenino $96(84,2 \%)$ y masculino $18(16 \%)$. La edad media fue $36 \pm 14$ años. Procedían del Departamento Central 42(42\%) e interior 59(58\%).

El tiempo de enfermedad promedio del LES fue de $5 \pm 6$ años. La media media de prednisona fue $25 \mathrm{mg} /$ día. Presentaron en el momento del estudio LES activo 40 pacientes $(35,1 \%)$, nefritis lúpica 46 pacientes $(40,4 \%)$ y nefropatía crónica $11(10 \%)$ (Tabla 1$)$.

Tabla 1. Factores de riesgo cardiovascular relacionados con el LES en pacientes atendidos en el Hospital Nacional (n 114)

\begin{tabular}{lcc}
\hline \multicolumn{1}{c}{ Factores de riesgo } & Frecuencia & Porcentaje \\
\hline Nefritis lúpica & 46 & 40,4 \\
Nefritis lúpica con hipertensión arterial & 24 & 52,1 \\
Nefritis lúpica con colesterol >200 & 17 & 36,9 \\
Lupus activo & 40 & 35,1 \\
Hipercomplementemia & 5 & 4,4 \\
Anticuerpo anti cardiolipina (+) & 1 & 0,9 \\
Nefropatía crónica & 11 & 10 \\
\hline
\end{tabular}

Los principales factores de riesgo cardiovascular tradicionales observados en nuestra población de estudio fueron: hipertensión arterial, el sobrepeso, 
obesidad, HDL bajo (Tabla 2).

Tabla 2. Factores de riesgo cardiovascular tradicionales asociados en pacientes con LES ( $n$ 114).

\begin{tabular}{lcc}
\hline \multicolumn{1}{c}{ Factores de riesgo } & Frecuencia & Porcentaje \\
\hline Hipertensión arterial & 47 & 41 \\
Sedentarismo & 52 & 45 \\
Colesterol HDL bajo & 44 & 38 \\
Sobrepeso & 43 & 37 \\
Obesidad & 18 & 15 \\
Hipertrigliceridemia & 24 & 21 \\
Hipercolesterolemia & 38 & 33 \\
Colesterol LDL $\geq 150$ & 6 & 5 \\
Circunferencia de cintura en $\mathrm{cm} \geq 88$ en mujeres & 37 & 32 \\
Circunferencia de cintura en $\mathrm{cm} \geq 102$ en varones & 4 & 3,5 \\
Síndrome Metabólico & 2 & 1,7 \\
Diabetes mellitus & 2 & 1,7 \\
Tabaquismo & 3 & 2,6 \\
\hline
\end{tabular}

Presentaron hallazgos del electrocardiograma con hipertrofia del ventrículo izquierdo compatibles con cardiopatía hipertensiva (Tabla 3).

Tabla 3. Hallazgos del electrocardiograma en pacientes con LES (n 114)

\begin{tabular}{lcc}
\hline $\begin{array}{c}\text { Hallazgos del } \\
\text { electrocardiograma }\end{array}$ & Frecuencia & Porcentaje \\
\hline Hipertrofia ventricular izquierda & 4 & 3,5 \\
Bigeminismo & 1 & 0,8 \\
\hline
\end{tabular}

Los hallazgos anormales de la ecocardiografía fueron sobre todo con cardiopatía hipertensiva (Tabla 4).

Tabla 4. Hallazgos ecocardiográficos en pacientes con LES ( $n$ 114)

\begin{tabular}{lcc}
\hline \multicolumn{1}{c}{ Hallazgos } & Frecuencia & Porcentaje \\
\hline Hipertrofia del septum & 6 & 5,2 \\
Disfunción diastólica & $\underline{3}$ & $\underline{2,6}$ \\
\hline
\end{tabular}

Una sola paciente presentó un evento cardiovascular (accidente cerebrovascular isquémico). Los hallazgos de la ecografía Doppler de carótida fue normal en todos los pacientes.

Una sola paciente presentó anticuerpo anti cardiolipina positivo (1\%). La dosis promedio de corticoides en nuestra serie fue de $25 \mathrm{mg} /$ día y todos recibieron hidroxicloroquina.

No se halló diferencia significativa cuando se comparó la presencia de HDL bajo, el sobrepeso u obesidad de las pacientes con el sedentarismo. La presencia de nefropatía crónica no se relacionó con la presencia de HTA. Pero cuando se comparó nefritis lúpica con la presencia de HTA fue estadísticamente significativo (Tabla 5). 
Tabla 5. Relación entre los factores de riesgo cardiovascular y su significancia estadística en pacientes con LES ( $\mathrm{n}$ 114)

\begin{tabular}{llc}
\hline \multicolumn{1}{c}{ Factores de riesgo } & Factores de riesgo & Valor $\mathbf{p}$ \\
\hline HDL bajo & Sedentarismo & 0,1 \\
Sobrepeso / obesidad & Sedentarismo & 0,1 \\
Sobrepeso / obesidad & Dosis de prednisona & 0,1 \\
HTA/ HTA no controladaHipertrofia ventricular & 0,6 \\
\multicolumn{2}{l}{$\begin{array}{l}\text { izquierda } \\
\text { Nefropatía crónica }\end{array}$ HTA } \\
\hline
\end{tabular}

\section{DISCUSIÓN}

La edad media de nuestra población fue de 36 años, en su mayoría mujeres, procedentes sobre todo del Departamento Central por ser el área de influencia de nuestro Hospital. El LES afectó sobre todo a mujeres jóvenes como pudo observarse en nuestro estudio.

Con relación a los factores de riesgo cardiovasculares asociado al LES: en la muestra presentaron LES activo 40 pacientes $(35,1 \%)$ a pesar del tratamiento con inmunosupresores, lo cual constituye un factor de riesgo cardiovascular para estos pacientes. Se sabe que la propia actividad inflamatoria de la enfermedad se asocia con un mayor riesgo cardiovascular como ha sido reportado por varios autores $(8,9,14)$.

Se puede constatar una relación altamente significativa entre el riesgo vascular y la actividad lúpica. Múltiples células del sistema inmunitario están presentes tempranamente en las lesiones ateroscleróticas, sus moléculas efectoras aceleran la progresión de las lesiones y la activación de mecanismos inflamatorios que vinculan fuertemente el desarrollo de eventos cardiovasculares(3). Esta asociación ha sido cuantificada y se estima que un incremento de 6 puntos en el índice SLEDAI durante un año se correlaciona con un aumento del $5 \%$ en el RCV a 2 años(8).

Una de las manifestaciones clínicas más frecuentes de la actividad inflamatoria del LES fue la nefritis lúpica, que se presentó en 46 pacientes $(40,4 \%)$, que ha sido asociada con una aterosclerosis acelerada y explica que la principal causa de mortalidad en pacientes con nefritis lúpica, hasta el $50 \%$ de los casos, sea la enfermedad cardiovascular o la enfermedad cerebrovascular ${ }^{(8)}$.

Otros factores involucrados en la nefritis lúpica para el desarrollo de la aterosclerosis acelerada son una mayor tendencia a la dislipidemia y a la HTA ${ }^{(8)}$. En nuestro estudio presentaron HTA y nefritis lúpica 24 pacientes $(52,1 \%)$ y la hipercolesterolemia asociada a la nefritis se observó en 17 $(36,9 \%)$. En esta muestra la presencia de nefritis lúpica fue estadísticamente significativo con el desarrollo de HTA. El uso amplio de corticoides y la enfermedad renal son factores que pueden explicar esta frecuente comorbilidad(23).

En cuanto al tiempo de enfermedad, el promedio fue de 5 años en la muestra, que está relacionada con una arteriosclerosis acelerada por la actividad de la enfermedad (22).

La dosis promedio de prednisona que recibían nuestros pacientes fue de $25 \mathrm{mg} /$ día. Aunque loscorticoides siguen siendo una de las terapias más efectivas para el manejo de la actividad en el LES, tienen un gran número de efectos metabólicos secundarios, tales como el aumento de la presión 
arterial, la glucosa, los lípidos y el peso. Se ha comprobado que una mayor duración de la corticoterapia se asocia con enfermedad cardiovascular subclínica e independientemente predice episodios cardiovasculares en pacientes con $\operatorname{LES}(7,8)$.

Los pacientes que reciben glucocorticoides, debido a que incrementan las cifras tensionales, presentan resistencia a la insulina y aumentan el riesgo cardiovascular (21). En nuestra muestra todos los pacientes recibían tratamiento con corticoides. No se tiene el perfil lipídico previo al uso de este fármaco, no se puede saber si la dislipidemia observada sea secundaria al uso de corticoides. Por ello sería de buena práctica conocer el perfil lipídico previo de los pacientes, porque se sabe que los corticoides pueden desencadenar o agravar una dislipidemia previa( ${ }^{(4)}$.

En cambio, la hidroxicloroquina ha demostrado efectos cardiovasculares beneficiosos en pacientes con LES, siendo hoy en día un tratamiento de primera línea. Se ha comprobado que reduce los niveles de colesterol total, CLDL, así como triglicéridos, y que incluso podría evitar la dislipidemia asociada a corticoides(7-9). En nuestro estudio todos los pacientes recibían hidroxicloroquina ${ }^{(4)}$.

Con relación a la nefropatía crónica, 11 pacientes presentaron esta complicación. Se sabe que en la insuficiencia renal, la hipertrigliceridemia es frecuente, la lipólisis disminuida de triglicéridos por la lipoproteinlipasa y una disminución en el clearance plasmático de lipoproteínas, son la principal causa de hiperlipemia en la insuficiencia renal( ${ }^{(4)}$. Además, se han encontrado anticuerpos antiproteinlipasa en el LES, responsabilizándolos de la hipertrigliceridemia que acompaña a esta enfermedad(23).

Como se ha visto, los pacientes con LES presentan mayor frecuencia de factores de riesgo cardiovascular tradicionales. Presentan dislipidemia con un perfil lipídico aterogénico denominado patrón lúpico de dislipoproteinemia, caracterizado por niveles elevados de colesterol total, triglicéridos, lipoproteína de baja densidad (c-LDL), y lipoproteína A, así como niveles disminuidos de lipoproteína de alta densidad (c-HDL). Los pacientes con LES también presentan con mayor frecuencia diabetes mellitus debido a la disminución significativa de la sensibilidad a la insulina y a la alta prevalencia de síndrome metabólico(21,22)

De igual manera, tienen mayor frecuencia de HTA, tabaquismo y sedentarismo(22). El sedentarismo se observó en el $52 \%$ de los pacientes, lo que es elevado; en otro estudio fue encontrado hasta en $70 \%$ de LES ${ }^{(23)}$. Se relaciona a una disminución de la capacidad aeróbica, disminución de la capacidad de ejercicio y fatiga en estos pacientes ${ }^{(23)}$.

Entre los factores de riesgo tradicionales, los principales factores identificados en nuestra población fueron la hipertensión arterial, el HDL bajo (31\%), hipertrigliceridemia, hipercolesterolemia, promedio de cintura de $88 \mathrm{~cm}$ en las mujeres, el sedentarismo, el sobrepeso y la obesidad que son factores de riesgo modificables con un tratamiento y medidas higiénico dietéticas oportunas. La obesidad fue encontrada en otros estudios en $20 \%$ a $56 \%$ de los pacientes con LES, se lo relaciona con el sedentarismo, la corticoterapia y la insulinorresistencia. Para algunos autores es un factor de riesgo independiente para el desarrollo de lesiones ateroscleróticas en el LES, hallándose que contribuye de forma independiente al aumento de marcadores inflamatorios en esta población ${ }^{(23)}$. 
Con relación al síndrome metabólico, la frecuencia del síndrome fue baja (2\%). Se presentó en mujeres, sin embargo, como fue muy baja no se pudo demostrar la asociación con la actividad de la enfermedad, dosis de prednisona y la duración de la enfermedad como lo demostraron Parker y colaboradores(19). La escasa observación del síndrome metabólico en nuestra serie podría deberse al tamaño de la muestra. Varios estudios mostraron que en pacientes con diagnóstico de LES existe una alta prevalencia de síndrome metabólico (16-18). Se necesitaría un mayor número de pacientes, lo cual constituye una limitante de nuestro trabajo. La obesidad abdominal y la actividad inflamatoria se mostraron como factores predictores de insulinorresistencia, al igual que la utilización de glucocorticoides ${ }^{(23)}$.

Igualmente, la frecuencia de diabetes mellitus y el tabaquismo fue baja en nuestra muestra. Se encuentra diabetes mellitus hasta en $5 \%$ de los pacientes con LES. La relación entre la insulinorresistencia y el LES podría explicarse por el estado inflamatorio crónico. Se ha descripto la presencia de anticuerpos antirreceptor de insulina en pacientes con LES, pero su prevalencia es baja(23).

Se ha reportado que aproximadamente el $25 \%$ de las muertes en LES se deben a afecciones cardiovasculares relacionadas con dislipidemias, elevación del IMC, resistencia a la insulina e HTA(16-19). La dislipidemia, el sobrepeso y la obesidad estuvieron presentes en nuestros pacientes.

En nuestro estudio cuando se comparó la presencia de HDL bajo, el sobrepeso u obesidad de las pacientes con el sedentarismo y no fue estadísticamente significativo. La presencia de nefropatía crónica no fue estadísticamente significativo con la presencia de HTA. Este resultado podría interpretarse como una limitación del estudio pues se trata de un diseño trasversal, se necesitaría estudios con un seguimiento más prolongado de esta población para obtener resultados más fidedignos.

En los pacientes con LES es esencial identificar la enfermedad cardiovascular de modo precoz para mejorar su manejo y así aumentar su supervivencia. En la última década se han desarrollado numerosas técnicas de imagen, cada vez más precisas, con las que podemos reconocer de una forma temprana los signos de enfermedad cardiovascular en pacientes asintomáticos $(7,8)$. Mediante estas técnicas, como la ecografía Doppler de carótidas, podemos valorar alteraciones de la función endotelial y modificaciones estructurales de la pared arterial como expresión de una aterosclerosis precoz. Los resultados de estas técnicas, junto con la valoración de los factores de riesgo cardiovascular, nos permitirán una mejor estimación y estratificación individualizada del riesgo y de este modo establecer una estrategia terapéutica adecuada para reducirlo(15).

Roman et al. publicaron un estudio en el que valoraron la prevalencia de la aterosclerosis subclínica y su relación con los factores relacionados con el LES. Estos autores encontraron una menor frecuencia de anticuerpos anti-Sm, anti-RNP y anticardiolipina en pacientes sin placas carotídeas y aquellos pacientes lúpicos con placas eran sobre todo pacientes caucásicos, de mayor edad e hipertensos(15). En nuestra serie ningún paciente presentó placas de ateroma a la ecografía Doppler de carótidas y solo un paciente presentó anticuerpo anti cardiolipina. No hay consenso sobre el papel de los anticuerpos antifosfolipídicos en el desarrollo de 
lesiones ateroscleróticas clínicas o subclínicas. Algunos investigadores encuentran relación entre los mismos y el desarrollo de lesiones arterioscleróticas en el LES, mientras que otros no hallan una relación clara entre ambos fenómenos ${ }^{(23,24)}$.

Con relación al anticoagulante lúpico se sabe que aumenta el riesgo cardiovascular al asociarse con mayores eventos trombóticos y mayor riesgo de infarto agudo de miocardio, los cuales se incrementan con la presencia de otros factores de riesgo cardiovascular (21).

En cuanto a la lesión de órgano blanco, sobre todo en los pacientes con HTA, muy pocos pacientes presentaron lesión de órgano blanco (hipertrofia del ventrículo izquierdo) tanto en el electrocardiograma como de la ecocardiografía. Este incremento es independiente de las cifras de HTA y se constituye en un riesgo cardiovascular aislado(23).

Una sola paciente presentó un evento cerebrovascular, un accidente cerebrovascular isquémico relacionado con la presencia de anticuerpos antifosfolipídico. Los pacientes con LES tienen mayor riesgo de ictus comparado con la población general el cual es atribuido a arteriosclerosis temprana, coagulopatía, vasculitis, embolismos cardiogénicos. La presencia de anticuerpos anticardiolipina se han asociado con estos eventos, además de los factores de riesgo independientes del LES (edad, HTA, diabetes mellitus), la actividad y la duración del LES(24).

En los pacientes con LES, la evidencia sugiere que existe una compleja interacción entre las alteraciones del sistema inmune de estos pacientes, la inflamación, los factores de riesgo tradicionales, la disfunción de células endoteliales y la reparación vascular, así como con algunos de los tratamientos usados para el control del LES. Todo ello ha conducido a que numerosos expertos consideren el LES como un factor de riesgo cardiovascular independiente. La conducta a seguir con relación a la evaluación del riesgo no es satisfactorio en estos pacientes debido a la falta de guías diseñadas para este grupo de pacientes $(7,8)$ que hace que sistemas como la escala de riesgo Framingham no refleje correctamente el RCV en estos pacientes. Esta situación se debe a la presencia de factores de RCV no tradicionales, los cuales no forman parte de las variables estudiadas en estas escalas. Por tal motivo la European League Against Rheumatism recomienda multiplicar por 1,5 el valor de riesgo cardiovascular en pacientes con LES(21, 22, 24).

Los resultados de esta muestra revelan que los principales factores de riesgo relacionados al LES identificados fueron la presencia de actividad de la enfermedad, la nefritis lúpica y la nefropatía crónica. Los principales factores de riesgo tradicionales identificados fueron la HTA, el sedentarismo, el sobrepeso/obesidad, c-HDL bajo, hipercolesterolemia e hipertrigliceridemia. Esto pone en evidencia un mayor riesgo de enfermedad cardiovascular, por lo que es necesario realizar el screening sistemático de los factores de riesgo cardiovascular y la búsqueda de arterosclerosis subclínica deben estar dentro de la rutina de seguimiento de esta población ${ }^{(23,24)}$.

Un control estricto de la actividad de la enfermedad y de los factores de riesgo cardiovasculares asociados, la mayoría de ellas factores de riesgo modificables, que, con un mejor control de la actividad de la enfermedad, cambios en el estilo de vida se podrían disminuir los eventos cardiovasculares en los pacientes con LES. 
Las limitaciones de este estudio: se trata de estudio trasversal, se necesitaría un seguimiento de estos pacientes y un aumento de la muestra para detectar las lesiones subclínicas que podrían ayudar a tomar las medidas preventivas oportunas y disminuir los eventos cardiovasculares en los pacientes con LES.

\section{REFERENCIAS}

1. Pérez Gómez A, Ruiz Gutiérrez, M. Sánchez A, Quintana. C. Lupus eritematoso sistémico (I) .Medicine. 2013;11(32):1955-65

2. Rahman A, Isenberg DA. Systemic Lupus Erythematosus. N Engl J Med. 2008;358(9): 929-939

3. Pérez $M$, Pérez $A$, Vázquez Gómez L. Factores asociados al riesgo vascular en pacientes con lupus eritematoso sistémico. Acta Médica del Centro.2013;7(4):1-10.

4. Calderón-Ticona J, Garro García D, Calvo A, Frecuencia dedislipidemia en pacientes con lupus sistémico. SPMI. 15(1):1-7.

5. Navarro M, Martínez G, Silva S, Pérez-Ybarra L, Ruíz M, López M. Factores de riesgo cardiovascular en pacientes con lupus eritematososistémico. ODOUS Cientifica. 2011;12(1):14-20

6. Villa-Forte A, Mandell BF. Trastornos cardiovasculares y enfermedad reumática. Rev Esp Cardiol. 2011;64(9):809-817

7. Magro-Checa C, Salvatierra J, Rosales J, Raya Álvarez E. Riesgo cardiovascular en el lupus eritematoso sistémico: factores implicados y métodos para su valoración. Semin Fund Esp Reumatol. 2012;13(3):95102

8. Ross R. Atherosclerosis-an inflammatory disease. N Engl J Med.1999;340(2):115-26.

9. Chung CP, Oeser A, Avalos I, Raggi P, Stein CM. Cardiovascular risk scores and the presence of subclinical coronary artery atherosclerosis in women with systemic lupus erythematosus. Lupus. 2006;15(9):562-9.

10. Calderón-González C, García-Fernández N. Enfermedad renal crónica:clasificación, etiopatogenia y factores pronósticos. Medicine. 2011;10(79):5364-9

11. James P, Oppari S, Carter BL, Cushman WC, Dennison-Himmelfarb C, Handler, et al. 2014 evidence-based guideline forthe management of high blood pressure in adults: report from the panel members appointed to the Eighth Joint National Committee (JNC 8).JAMA. 2014;311(5):507-20.

12.Rodríguez S, Mas P, Frühbeck Martínez G. Obesidad, concepto, clasificación, implicaciones fisiopatológicas, Complicaciones asociadas. Medicine 2004, 9(19):1167-75

13. Zimmet P, Alberti G, Shaw J. Nueva definición mundial de la FID del síndromemetabólico. Diabetes. 2005;50(3):31-33

14. Calmarza Calmarzaa P, Civeira Murillob F. Protocolo diagnóstico de las dislipidemias. Medicine. 2013;11(40):2424-8

15. Roman MJ, Shanker BA, Davis A, Lockshin MD, Sammaritano L, SimantovR, et al. Prevalence and correlates of accelerated atherosclerosis in systemic lupus erythematosus. N Engl J Med. 2003;349(25):2399-406 16. Expert Panel on Detection, Evaluation, and Treatment of High Blood Cholesterol in Adults. Executive summary of the third report of the National Cholesterol Education Program (NCEP) Expert Panel on Detection, Evaluation, and Treatment of High Blood Cholesterol in Adults (Adult Treatment 
Panel III). JAMA. 2001;285(24):86-97.

17. Romero-Moreno JR, Ramírez-Villafaña M, González-Ponce F, Díaz-Rizo C, Araújo-López A, Rodríguez-Hernández T, et al. Síndrome metabólico en lupus eritematoso sistémico. El Residente. 2014;9(3):116-123

18. Parker B, Bruce IN. The metabolic syndrome in systemic lupus erythematosus. Rheum Dis Clin North Am. 2010;36(1):81-97

19- Parker B, Urowitz MB, Gladman DD, Lunt M , Bae SC, SanchezGuerrero J, et al. Clinical associations of the metabolic syndrome in systemic lupus erythematosus: data from an international inception cohort. Ann Rheum Dis. 2013;72(8):1308-1314.

20. El Magadmi M, Ahmad Y, Turkie W, Yates AP, Sheikh N, Bernstein RM, et al. Hyperinsulinemia, insulin resis-tance, and circulcating oxidized low density lipoprotein in women with systemic lupus erythematosus. J Rheumatol. 2006;33(1):50-56

21. Batún Garrido JA, Radillo $H$, Hernández Núnez AE. Riesgo cardiovascular en lupus eritematoso sistémico. Rev Colomb Reumatol. $2016 ; 23(4): 242-249$

22. Batún Garrido JA, Radillo $H$, HernándezNuñez AE. Riesgo cardiovascular en lupus eritematoso sistémico. Rev Colomb Reumatol. $2016 ; 23(4): 242-249$

23. SilvariñoR, Sato E. Factores de riesgo para aterosclerosis en enfermedades autoinmunitarias sistémicas. Rev Med Urug. $2008 ; 24(2): 118-132$

24. Diaz Cortez D, Correa Gonzalez N, Diaz MC, Gutierrez JM, Fernandez Avila D. Compromiso del sistema nervioso centralen el lupus eritematoso sistemico. Rev Colomb Reumatol. 2015;22(1)16-30 